\title{
Teorías constructivistas, perspectiva funcional-estructural en el aprendizaje del fútbol base Constructivist theories, functional-structural perspective in learning in initiation to football \\ *Sagrario del Valle Díaz, *Manuel Antonio Rubio Palomino, *Jose María Nevado Luna \\ *Universidad de Castilla La Mancha (España)
}

Resumen: Se presenta una revisión teórica de los estudios que se han realizado en fútbol desde el paradigma cognitivo. El artículo pretende argumentar la necesidad de distinguir bajo el paradigma cognitivo, dos líneas de trabajo diferentes ya que se pretende desarrollar la toma de conciencia en las acciones de juego. No es lo mismo enfocar un entrenamiento bajo las teorías del procesamiento de la información (perspectiva estructural-funcional), que bajo las teorías constructivistas (perspectiva funcional-estructural), centrando el interés en el conocimiento previo del jugador, el desarrollo evolutivo, la categoría y los distintos niveles de pensamiento. Por tanto se pretende bajo el paradigma cognitivo, teorías constructivistas, perspectiva funcional-estructural, dar respuesta a un modelo de aprendizaje en fútbol base eficaz, efectivo y eficiente, transferible a cualquier deporte de invasión.

Palabras clave: fútbol, paradigma cognitivo, teorías constructivistas, pensamiento.

\begin{abstract}
A theoretical review was carried out in football under the cognitive paradigm. The article aims at demonstrating the need to distinguish two different lines of work under the cognitive paradigm as we try and develop awareness in game actions. It is very different to build a training through the framework of the theories of information processing (structural-functional perspective), or through constructivist theories (functional-structural perspective), which focus on players' knowledge, development, division, and different levels of awareness. Thus, within the cognitive paradigm, constructivist theories, and functional-structural perspective, we intend to respond to an effective, efficacious, and efficient initiation-to-football training model, transferable to any invasion sport.
\end{abstract}

Key words: football, cognitive paradigm, constructivist theories, awareness.

\section{Introducción}

El artículo muestra una nueva tendencia en el proceso de aprendizaje del fútbol base desde el paradigma cognitivo, teorías constructivistas, perspectiva funcional-estructural. Nos interesa resaltar que en el aprendizaje «experimentar por experimentar» no genera diferentes niveles de pensamiento en el futbolista, si lo que pretendemos es que adquiera pericia que es diferente a la suma de experiencia.

Han proliferado muchos modelos que persiguen el desarrollo de un futbolista integral desde el paradigma cognitivo, pero son pocos los estudios que evidencian la diferencia entre el uso de las teorías del procesamiento de la información o el uso de las teorías constructivistas, para obtener un mayor rendimiento en el aprendizaje (Arda \& Casal, 2003; Corbeau, 1990; Fradua, 2005; García Aliaga, 2005; Garganta \& Pinto, 1997; Lago, 2001, 2002, 2007; Martínez Garfía \& Sáenz-López, 2000; Moreno \& Morcillo, 2004; Pacheco, 2004 y Seirul-lo, 2004).

Es por ello que la teoría del aprendizaje motor que aporta luz al aprendizaje en fútbol base, desde el paradigma cognitivo, debe prestar atención a la intencionalidad de las teorías que alumbran dicho proceso. No es lo mismo entrenar con un diseño de sesión amparado por las teorías del procesamiento de la información (perspectiva estructuralfuncional), que basar la sesión en las teorías constructivistas (perspectiva funcional-estructural).

La cuestión pivota sobre el eje de hallar en el mínimo tiempo el máximo rendimiento en el aprendizaje del futbolista que se inicia en el deporte, de modo que genere un esquema motor capaz de refinarse con alta eficacia, efectividad y eficiencia, durante su vida deportiva.

Establecer un marco teórico robusto en el aprendizaje del

Fecha recepción: 31-07-19. Fecha de aceptación: 03-02-20 Sagrario Del Valle Sagrariodel.Valle@uclm.es fútbol base daría la posibilidad de diseñar sesiones de entrenamiento con nuevas metodologías activas que permitan desarrollar el máximo rendimiento en el mínimo tiempo, acorde con la edad del futbolista, el desarrollo evolutivo, el conocimiento previo y los distintos niveles de toma de conciencia, en línea con lo que plantean (Del Valle \& De la Vega, 2008).

En definitiva se pretende reflexionar sobre el modo de generar aprendizajes significativos en el futbolista que se inicia, contrastando los niveles de pensamiento que construye en el entrenamiento, para ser más efectivo y a su vez, orientar a los entrenadores en las cuestiones que son clave para poder diseñar entrenamientos eficaces y de calidad.

\section{Estado actual del tema}

Se presenta una alternativa novedosa para el aprendizaje del fútbol base y por defecto aplicable a cualquier deporte de invasión, enfocado desde el paradigma cognitivo, teorías constructivistas, perspectiva funcional-estructural, cuyo objetivo es construir un modelo que dé respuesta a la adquisición de un aprendizaje significativo y eficaz en el deporte.

Como señala Karmiloff-Smith (1994), si quieres avanzar hazte con una teoría. Pues bien, este es nuestro interés, proponer un marco teórico que pueda alumbrar el saber práctico del aprendizaje del fútbol base y su mejora.

Por ello, de cara a establecer la línea argumental dentro del aprendizaje significativo y eficaz en fútbol base, nos interesa desarrollar en el futbolista que aprende unos patrones de movimiento que una lo que piensa con lo que hace, para que se produzca una toma de decisión robusta, capaz de ser generalizada y automatizada con la mayor eficacia posible, en el mínimo tiempo.

De ahí que en línea con Garganta \& Cunha (2000) podemos defender que siendo el juego del fútbol una secuencia de secuencias, implica que los jugadores tienen 
que estar en condiciones de inventar nuevos juegos durante el juego, siendo en las zonas de incertidumbre en las que se determinan las fases críticas. Aspectos que hacen referencia al pensamiento con carácter de uso en los futbolistas, necesario saberlo desarrollar en las etapas de formación. $\mathrm{O}$ cuando defienden dichos autores que la creatividad y lo inesperado está relacionado con la capacidad de, a partir de las acciones ambiguas y simulaciones, crear sorpresa en el adversario. Así la cuestión se centra en cómo generar el pensamiento y la creatividad, sin dar por hecho que por la mera experimentación el jugador lo desarrolla. Claro que para ello es necesario diseñar sesiones que generen un conflicto cognitivo al futbolista para que aprenda a resolverlo poniendo en juego diferentes niveles de pensamiento.

En la misma línea hallamos a Vegas Haro (2006) que señala que para inventar juegos nuevos, como para plantear o generar estrategias, así como para crear nuevas soluciones, se hace necesario poseer un bagaje de conocimientos acerca del área que nos movemos, en pos de desarrollar un pensamiento resolutivo creativo y eficaz. Lo que nos hace pensar que es una condición necesaria pero no suficiente.

Bajo este telón de fondo, es perentorio crear en los jugadores un conocimiento práctico con la mayor calidad posible. Para generar dicho saber práctico es necesario poner el pensamiento en acción y uno de los caminos posibles sería dejarse alumbrar por el paradigma cognitivo, las teorías constructivistas y la perspectiva funcional-estructural, que asume en su planteamiento teórico lo que estamos poniendo de manifiesto. Veamos a qué nos referimos.

El fútbol es un deporte que ha evolucionado en el aprendizaje desde un paradigma conductista a un incipiente paradigma cognitivo. En la enseñanza del fútbol, aunque se siguen aplicando estrategias conductistas basadas en el aprendizaje de la repetición de la técnica de una forma mecanicista, se observa cómo se atiende a la complejidad de las situaciones reales de juego donde se pone de manifiesto otro tipo de planteamiento. Aunque en muchas ocasiones actualmente nos encontramos que en el aprendizaje del fútbol base todavía se hallan entrenamientos cuyos parámetros de condición física, técnica y táctica se organizan y enseñan de manera repetitiva, como elementos externos al juego y sin ningún proceso cognitivo.

Por ejemplo encontramos clasificaciones de autores como Lealli (1994), Sampedro (2000), Pacheco (2004), Benedek (2001), Sans Torrelles y Frattarola (1999), incluso Brüggemann (2004), que proponen líneas de acción basadas en las características generales de cada etapa del jugador, en las que se describen distintas relaciones a seguir en los entrenamientos de fútbol como: relación jugador-balón (prebenjamín), relación jugador-adversario (benjamín), relación jugador y compañeros (alevín), relación jugadorequipo (infantil), relación jugador-equipo-rival (cadete); $\sin$ embargo no nos aportan herramientas ni contenidos propios del fútbol, suficientes para adentrarnos en el proceso de aprendizaje si tratamos de analizar el pensamiento del jugador; por lo que no hay un marco teórico definido en donde sustentar el conocimiento práctico.

Cada vez más hay que apostar por otro tipo de entrenamiento más contextualizado, basado en un paradigma cognitivo donde el modelo teórico sea evidente, por ejemplo: no es lo mismo centrarse en la teoría del procesamiento de la información, perspectiva estructural-funcional, en donde se da mucha importancia a los mecanismos de percepción, decisión y ejecución. Se estudia la interpretación, el procesamiento y almacenamiento de las acciones de juego en la memoria. El futbolista tiene un desarrollo cognitivo donde el aprendizaje está relacionado con las acciones que realiza para resolver los problemas que le plantea el juego, asumiendo que el futbolista al tomar decisiones piensa, sin atender a la naturaleza intrínseca del pensamiento per sé.

De hecho se entiende dicha perspectiva estructuralfuncional como el análisis de los movimientos del sujeto para entender formalmente la actuación como estructuras articuladas que no excluyen una función concreta, pero sí desatienden el proceso por el cual el futbolista construye su propio pensamiento o, concretamente, se desatiende la construcción epistémica de los diferentes niveles de toma de conciencia (Del Valle \& De la Vega, 2008).

Las propuestas que defienden el procesamiento de la información se describen como operaciones de planificación, ejecución y control de la totalidad de las actividades perceptivo-motrices que se dan o no, asumiendo sin contrastarlo que el futbolista piensa. En dicho postulado la memoria, la atención, la retroalimentación sensorial y la motivación son determinantes. Las medidas de evaluación más comunes en la investigación son por ejemplo: el tiempo de reacción, medidas de error, tiempo de ejecución (Del Valle etal., 2008).

Autores desde teorías del procesamiento de la información como Allard, Graham \& Paarsalu (1980), Suárez \& Hernández (2007), exponen que en baloncesto y en los deportes de invasión los expertos tienen mayor capacidad para estructurar y organizar la información. En la misma línea en voleibol hallamos el estudio de Gil, Del Villar, Claver, Moreno, García, Moreno (2012), donde llegan a la conclusión de que los jugadores que participan a nivel nacional, respecto a los de nivel regional y escolar, son los que mayor conocimiento declarativo y procedimental muestran; claro que los autores no profundizan en los tipos de pensamiento que construyen o en niveles de toma de conciencia que desarrollan. Bard \& Fleury (1981) en Hockey señalan que los expertos pueden predecir las trayectorias de los objetos. Si nos damos cuenta parece que hay que llegar a ser expertos para obtener niveles de pensamiento eficaces. La cuestión es con qué comparar la información de la investigación. No es lo mismo comparar resultados con los datos hallados en un estudio con planteamiento conductista que cognitivo, y dentro del cognitivo, si proviene desde la teoría del procesamiento de la información o de las teorías constructivistas. Incluso ir más allá y trabajar asumiendo que el sujeto piensa desde la teoría ecológica y el planteamiento de la pedagogía no lineal en relación con lo que plantean Cantos \& Moreno (2019), aplicado en rugby. En fútbol se tiene en cuenta las características generales de cada etapa del jugador y se introducen elementos técnicostácticos propios del deporte como son: roles de juego, acciones técnico-tácticas... Este marco práctico sigue mostrando una serie de características generales, sin plasmar una estrategia definida de enseñanza de los conceptos que debe adquirir el futbolista, entrando en juego los niveles de 
pensamiento. Por lo que sigue sin mostrar un marco teórico de naturaleza funcional para ser eficaz y eficiente en el juego. Autores representativos son Arda y Casal (2003), Corbeau (1990), Fradua (2005), Garganta \& Pinto (1997), Lago (2001, 2002, 2007) Martínez Garfía \& Sáenz-López (2000), Moreno \& Morcillo(2004), GarcíaAliaga (2005), Pacheco(2004), Seirullo (2004) o Sierra-Olivares \& García-Rubio (2017) y SierraOlivares, Prieto-Ayuso, Pastor-Vicedo, \& González-Víllora (2020), que desde el paradigma cognitivo asumen incluso la teoría ecológica junto con el procesamiento de la información, aplicado al fútbol.

En este contexto teórico-práctico podemos ver como los autores se distancian de teorías conductistas sin mostrar cómo el futbolista piensa y toma soluciones en los problemas que ocurren en el terreno de juego de forma eficaz, cayendo en el error de que experimentar por experimentar provoca aprendizaje.

La cuestión es plantearse si es posible llegar a ser experto en la iniciación deportiva y cómo, sin olvidar que las teorías del procesamiento de la información no explican, ni entienden el proceso por el cual el deportista analiza la acción y toma una decisión. Simplemente se centran en el jugador que procesa una acción que tiene en la memoria y la ejecuta como una computadora sin que exista relación entre el sujeto y el medio. Se asume la importancia de la reflexión sobre la acción, pero no se profundiza en cómo se construye a nivel funcional la toma de conciencia.

El problema por tanto, se plantea en las teorías del procesamiento de la información (la perspectiva estructuralfuncional), cuando se asume por imperativo categórico que el futbolista piensa y no se cae en la cuenta de que ¿y si no piensa? ¿Qué piensa? ¿A qué nivel toma conciencia? ¿Para qué piensa? ¿Experimentar por experimentar otorga al futbolista la posibilidad de encontrar la solución al problema con la máxima eficacia y en el mínimo tiempo?...

En fútbol Arda \& Casal (2003), Corbeau (1990), Fradua (2005), García Aliaga (2005), Garganta \& Pinto (1997), Lago (2001, 2002, 2007), Martínez Garfía \& Sáenz-López (2000), Moreno \& Morcillo (2004), Pacheco (2004) y Seirul-lo (2004), nos ofrecen modelos de entrenamiento y enseñanza del fútbol amparados en el paradigma cognitivo, pero cuando se profundiza en ellos se observa que se da por hecho que el futbolista piensa y no se explica cómo se generan los niveles de pensamiento en los jugadores. Por lo tanto aunque rompen con el conductismo y las teorías mecanicistas, se quedan inmersos en la teoría del procesamiento de la información, perspectiva estructural-funcional, dando por hecho que el futbolista piensa.

Es evidente que esperar o asumir que el futbolista piensa es perder evidencias claras de los resultados de aprendizaje y de la eficacia en el aprendizaje del fútbol base. Por tanto es necesario buscar otra alternativa dentro del paradigma cognitivo que articule otra perspectiva más efectiva. Nos referimos a establecer un marco teórico basado en el paradigma cognitivo, teorías constructivistas, perspectiva funcional-estructural, como alternativa a la teoría del procesamiento de la información.

La perspectiva funcional-estructural parte de la visión de que las acciones de los futbolistas suponen en general modificaciones ordenadas para cumplir determinados objetivos. Además estos autores se plantean tres problemas básicos a resolver aplicables al fútbol (Del Valle \& De la Vega, 2008):

1.¿Cómo se optimiza el orden de los movimientos a nivel funcional de forma reproducible, teniendo en cuenta las exigencias de la tarea y la experiencia? (problema del aprendizaje).

2.¿Por qué y hasta qué punto se eligen y se intentan unos movimientos organizados y no otros? (problema de la intencionalidad).

3.¿Hasta qué punto la reproducción y la intencionalidad presuponen una representación interna y cómo está constituida dicha representación?, ¿Cuál es su función y su estructura y qué problemas específicos aparecen en el sujeto en torno a dicha cuestión? (problema de la representación).

Así en fútbol nos encontramos con tres aspectos a tratar desde dicho planteamiento: el problema del aprendizaje, el problema de la intencionalidad y el problema de la representación que el futbolista construye cuando resuelve situaciones de juego.

De los tres aspectos citados anteriormente, por orden de importancia primero es necesario explicar qué ocurre respecto a la representación como elemento clave en el proceso de aprendizaje y en el uso de la intencionalidad del futbolista, que pretende resolver una situación de juego dada.

Por tanto, es necesario conocer cómo las representaciones (tipos de pensamiento), explican el acto motor, lo controlan o bien son elementos que construyen el conocimiento en fútbol.

Llegados a este punto, desde una perspectiva práctica, es complicado aclarar dónde empieza y dónde acaba lo funcional y lo estructural en una determinada acción en fútbol. La diferencia que podemos alumbrar es que en la perspectiva funcional-estructural hay una explicación epistemológica de cómo se origina el conocimiento, cómo es la naturaleza del pensamiento. Hecho relevante que es la clave diferenciadora con el otro postulado, que asume que el futbolista piensa sin explicar su naturaleza interna.

Según Zamora (2006) la teoría que da pie para soportar la perspectiva funcional-estructural de la representación, tiene como hipótesis de base que el sujeto construye el conocimiento a partir de su experiencia previa y mediante una interacción con el medio. Además esto nos muestra una idea clave: cada sujeto genera su propio conocimiento y es diferente al de los demás.

Con esta propuesta en línea con el autor anterior, nos situamos en el fútbol en un punto medio entre el empirismo y el racionalismo, ya que el futbolista en su aprendizaje y en situación real de juego, puede realizar representaciones mentales e interpretar lo que observa y hace en la realidad. Su conocimiento será el producto de dichas interpretaciones interiorizadas y relacionadas con la observación y su acción.

Según Piaget (1974) en la definición de un futbolista que aprehende la realidad como sujeto epistémico activo, con la conciencia del objetivo que pretende alcanzar y la conciencia del éxito o fracaso de su acción, conlleva que tanto los objetivos como los resultados sean conscientes en la acción intencional, aunque los medios usados para conseguirlo no lo sean. La intención surge del futbolista, pero el proceso de conocimiento nace de la interacción entre el futbolista y el 
objeto de conocimiento que son la resolución de las distintas situaciones a las que se enfrenta en el fútbol. En dicho acto de conocimiento el futbolista genera una representación que conlleva la comparación entre las distintas experiencias vividas con lo novedoso que pretende acometer. Por lo tanto genera una toma de conciencia que implica la atribución de significado a la acción. Único modo de que los procesos de la toma de decisión contribuyan a la autorregulación de la acción (Del Valle, 1999). Ya que si no hay autorregulación de la acción no hay posibilidad de construir nuevos y mejores aprendizajes; al menos desde un punto de vista práctico que sea eficaz, en cuanto a la comprobación de los resultados de aprendizaje.

Hay autores que empiezan a introducir esta línea de pensamiento en otros deportes como el baloncesto, por ejemplo Solá $(1998,2005)$, que trata de demostrar que la formación cognoscitiva previa a la acción táctica deportiva, es significativamente positiva para el desarrollo de la misma. No obstante autores como De la Vega (2002) en su estudio sobre diferentes niveles de comprensión táctica de un juego colectivo, Del Valle (1999) en gimnasia artística y Rychecki (2001) en judo, señalan que existen cambios en las representaciones tras tomar decisiones en las distintas acciones, demostrando que existen diferentes niveles de toma de conciencia cuando los deportistas actúan para mejorar la construcción de su aprendizaje y así dominar las acciones en el deporte.

Se pone de manifiesto que asumiendo las teorías constructivistas, perspectiva funcional-estructural, contemplamos una relación entre el futbolista y el juego que conlleva una toma de conciencia que viene dada por la construcción de la representación. Otra cuestión será establecer las estrategias de pensamiento que generan cada uno de los tipos de representación que debe construir el futbolista, según su desarrollo evolutivo, en función de las etapas del juego y cómo hacerlo. Todo ello directamente relacionado con la intencionalidad que el futbolista tiene para conseguir el objetivo, redundando en una mejora del aprendizaje y la eficacia.

Veamos entonces según el desarrollo evolutivo, qué tipo de representaciones construyen los niños en el juego del fútbol, para poder categorizar el aprendizaje del fútbol base, junto con la intencionalidad práctica.

Tomando como base la visión piagetiana (1974) en la que la toma de conciencia sobre la acción tiene un papel fundamental, podremos desvelar distintos niveles de conocimiento que se verán reflejados en las acciones que los futbolistas llevan a cabo, según el tipo de representación que construyan.

Tal y como citan Lacasa y Villuendas (1988) la importancia que da Piaget a la autoconciencia tiene sentido porque la persona tiene que generar sus propios procesos usando sus estructuras cognitivas. Este desarrollo se lleva a cabo por los procesos de asimilación, acomodación y equilibración.

En la relación del futbolista con el medio se forman los esquemas de acción, que Delval (1994) cita como «sucesiones de acciones materiales o mentales» que tiene una organización y que son susceptibles de repetirse en situaciones semejantes».

A partir de aquí podemos establecer varias ideas: a) La acción se produce antes que la representación; b) En los niveles superiores es la conceptualización la que dirige la acción. Este último punto es importante, ya que cuando el futbolista es capaz de invertir las fases y puede operar sobre las representaciones que construye, saliendo de un estado de equilibrio, y puede cambiar los límites impuestos en la realidad, tiene mayor capacidad de resolución.

Así pues, considerando la acción como una forma vital en la relación futbolista-medio, y separando el plano de la acción y el de la conceptualización, podemos distinguir distintos planos de conocimiento en el aprendizaje del fútbol base, siendo el primero una relación ensayo-error. En el segundo plano hay ya una toma de conciencia sobre la acción y podemos encontrar asimilaciones horizontales, pero no llegaremos al tercer plano hasta que seamos capaces de realizar operaciones formales denominadas también «abstracciones reflejas» Piaget (1976).

Siguiendo a De la Vega, (2002) distinguimos dos tipos de abstracción en el aprendizaje del fútbol: a) Empírica que se basa en hechos constatables ya que se puede comprobar la información de los datos del objeto o de la acción y b) Reflexiva en la que es necesario establecer relaciones que se alejan de lo que podemos comprobar empíricamente. La toma de conciencia se da sobre el plano de la acción. A su vez, dentro de ésta abstracción reflexiva podremos distinguir entre: $b .1)$ la inconsciente que surge por la comparación de elementos comunes; b.2) la abstracta que es capaz de acceder a sus propios conocimientos.

Por lo tanto parece ser necesario establecer una relación entre el desarrollo evolutivo del jugador de fútbol y el tipo de representación que construye sobre su acción. Esto es importante ya que el entrenador de fútbol tiene que entender el nivel cognitivo previo del que parte el futbolista y los diferentes niveles de toma de conciencia que construye en el aprendizaje del deporte. Hecho que muestra una visión funcional-estructural del proceso. Solo así se podrá controlar el resultado de aprendizaje y ser más eficaz en la enseñanza diseñando los entrenamientos.

De ahí que sea necesario conocer cómo se produce el desarrollo evolutivo de los futbolistas y los tipos de pensamiento que podemos desarrollar en los entrenamientos para aplicarlo con eficiencia, eficacia y efectividad, en el juego real.

\subsection{Desarrollo evolutivo de los futbolistas}

Respecto al desarrollo evolutivo del futbolista en relación con los tipos de representaciones que pueden llegar a construir, los niños de 2-6 a 8 años, tal y como cita Corral y Pardo (1997), pueden actuar inteligentemente salvo actos engañosos perceptivamente que pueden llevarles a equívoco. Es decir en estas edades habría que atender, en los entrenamientos, a los aspectos cuantitativos que entran en juego, como señala Vuyk (1981), porque los niños no pueden leer los aspectos observables. De ahí que cualquier comprensión de reglas de actuación en los entrenamientos debería incluir la orientación espacio-temporal (nociones topológicas claras, cuantificables), sumado a nociones de organización espacial (cuantificando distancias, intervalos y trayectorias), en progresión de dificultad en función de la edad. Teniendo en cuenta que hasta los 10 años no se tiene 
el componente global de la comprensión del juego por lo que hay que trabajar con no más de 3-4 nociones a la vez.

Superada la etapa de categoría prebenjamín-benjamín. En la etapa de alevines, tal y como vemos reflejado en Bueno, Del Valle, De la Vega, Velázquez, González \& Juárez (2014), pasada la etapa preoperacional se supera el egocentrismo y los jugadores ya pueden representar situaciones no sólo estáticas, sino que pueden construir habilidades para explicar personalmente sus representaciones mentales, operando sobre ellas. Aunque son incapaces de llegar a todos los resultados lógicamente posibles porque no acceden todavía a los conceptos abstractos. Cualquier comprensión de reglas de actuación en los entrenamientos debería incluir la orientación espacio-temporal (nociones topológicas claras, cuantificables), sumado a nociones de organización espacial (cuantificando distancias, intervalos y trayectorias), en progresión de dificultad en función de la edad, con predominio de estas últimas, ya que los jugadores a los 10 años adquieren el componente social del juego.

En la etapa operacional que comienza a los 11-12 años, etapa de infantil-cadete, según Bueno et al., (2014) se comienza a dominar la proporción y la conservación sistematizando operaciones complejas que llegan a todos los objetivos posibles. En este punto de desarrollo intelectual los futbolistas son capaces de llevar a cabo operaciones lógicoformales, se puede enfrentar a la resolución de sistemas simbólicos complejos. Momento adecuado para expandir el carácter reflexivo aprendiendo todos los contenidos del fútbol, generando niveles elevados de toma de conciencia. Aprendizaje significativo y eficaz. Cualquier comprensión de reglas de actuación en los entrenamientos debería incluir la orientación espacio-temporal, nociones de organización espacial (cuantificando distancias, intervalos y trayectorias) y la estructuración espacial (orientación más organización espacio-temporal), en progresión de dificultad.

Ahora bien cabe preguntarse: ¿Qué tipos de pensamientos tenemos que desarrollar en los futbolistas y qué estrategias podemos usar para poder generar procesos de pensamiento en los diferentes niveles de toma de conciencia, con el objetivo de ser eficaz en el aprendizaje y en el uso de la intencionalidad en el entrenamiento para transferirlo al juego real? Veamos la posible respuesta.

\subsection{Tipos de pensamiento a desarrollar en los futbolistas en los entrenamientos}

Swartz, Costa, Beyer, Reagan \& Kallick (2013) nos muestran que el pensamiento eficaz se refiere a la aplicación competente y estratégica de destrezas de pensamiento y hábitos de la mente productivos, que nos permite llevar a cabo actos meditados de pensamientos, como tomar decisiones, argumentar y otras acciones analíticas, creativas o críticas. En esta línea los autores señalan que el pensamiento eficaz está formado por: $1^{\circ}$ ) Destrezas de pensamiento que sería emplear procedimientos reflexivos específicos y apropiados para un ejercicio de pensamiento determinado (de acuerdo con los tipos de representación y niveles de toma de conciencia). $2^{\circ}$ ) Generar hábitos de la mente referido a conducir los procedimientos para dar lugar a conductas de reflexión amplias y productivas relacionadas con el hecho de pensar. $3^{\circ}$ ) Metaconocimiento que es usar los dos aspectos anteriormente citados basándose en la valoración que se hace de lo que se pide y de nuestro plan para llevarlo a cabo. Todo ello transferible completamente al fútbol.

En línea con lo expuesto para Swartz et al., (2013), existen diferentes tipos de pensamiento que deberíamos enseñar y que se deberían aplicar en los entrenamientos de fútbol. Ya que no vale únicamente la visión simplista de hacer preguntas reflexivas, ni tampoco el mero hecho de comparar y contrastar sin asegurarnos que se realiza de manera eficaz.

De ahí, los tipos de pensamiento que deberíamos provocar en fútbol para que sean eficaces, capaces de generar distintos niveles de toma de conciencia y por tanto, construir en el aprendizaje diferentes representaciones con distinta intencionalidad, son los mostrados en la (Tabla ${ }^{\circ} 1$ ), teniendo en cuenta el desarrollo evolutivo de los jugadores.

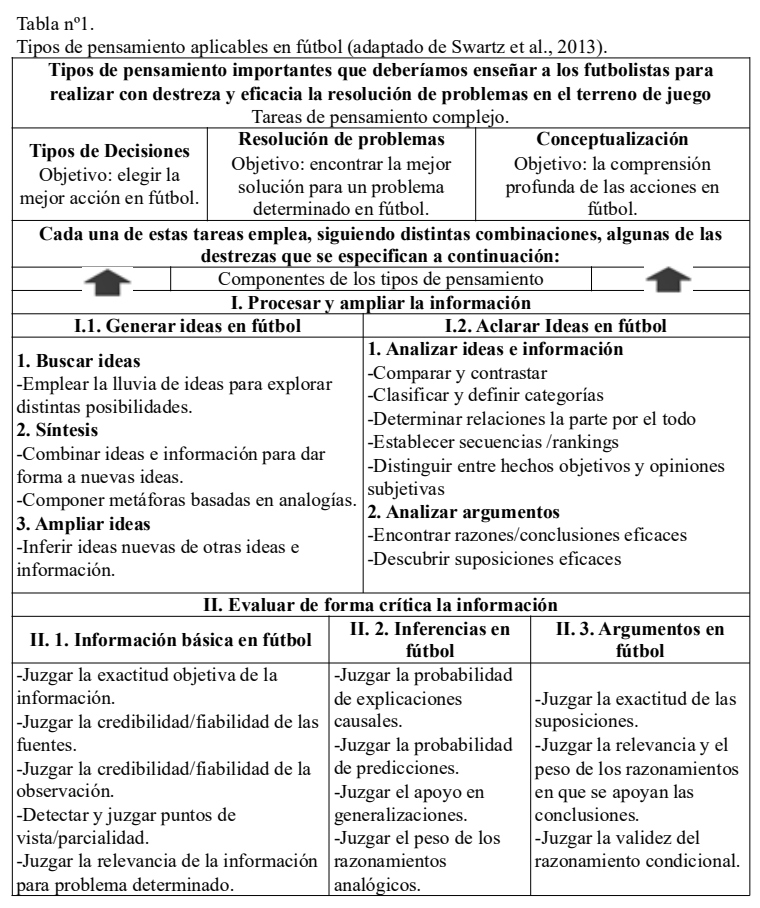

Así pues en fútbol base, en los entrenamientos, de cara a desarrollar una comprensión práctica del fútbol poniendo el pensamiento en juego, el entrenador tendrá que seleccionar por categorías, teniendo en cuenta el desarrollo evolutivo de los niños, los aspectos técnico-tácticos que quiera que aprendan los jugadores, junto con el tipo de pensamiento que necesita desarrollar, planteando jugadas que conlleven un conflicto cognitivo.

No es lo mismo centrar la atención en elegir la mejor acción en fútbol, que encontrar la mejor solución en fútbol o que generar una comprensión más profunda del fútbol. Todo ello aderezado con si se pretende que el futbolista procese la información y la amplíe o si se persigue que el futbolista evalúe de forma crítica la información (utilizando los diferentes componentes que se han mostrado).

Por tanto, la cuestión pivota sobre cómo confeccionar el entrenamiento en fútbol, según la categoría de juego, desarrollando el pensamiento de los futbolistas -sin olvidar el desarrollo evolutivo-, para lo que habría que utilizar distintas preguntas que concreten los tipos de pensamiento y hacer que expliciten el tipo de pensamiento que han construido los jugadores. 
Es importante que el jugador responda de manera concreta y con significado preciso a las cuestiones planteadas, tal y como afirma De la Vega (2002), para construir representaciones con distintos niveles de toma de conciencia, que articulen el aprendizaje eficaz en fútbol y dirijan la intencionalidad.

Para ello el entrenador tiene que dominar el contenido de enseñanza del fútbol y la metodología adecuada, además de poner en juego la motivación, para conseguir la meta propuesta.

\section{Discusión y conclusión}

El objetivo ha consistido en intentar establecer un marco teórico dentro del paradigma cognitivo, teorías constructivistas, perspectiva funcional-estructural en el aprendizaje del fútbol base, de tal forma que se puedan diseñar entrenamientos efectivos y eficaces, según la categoría de juego y el desarrollo evolutivo de los jugadores, para lograr diferentes niveles de pensamiento en los jugadores.

Para crear el contexto teórico de la forma en cómo genera pensamiento el futbolista cuando soluciona situaciones durante el juego, hay autores que siguen la línea del paradigma cognitivo, teoría del procesamiento de la información, perspectiva estructural-funcional como: Arda y Casal (2003), Corbeau (1990), Fradua (2005), García Aliaga (2005), Garganta \& Pinto (1997), Moreno \& Morcillo (2004), Lago (2001, 2002 y Lago, 2007), Martínez Garfía \& SáenzLópez (2000), Moreno \& Morcillo (2004), Pacheco (2004), Seirul-lo (2004), Sierra-Olivares \& García-Rubio, J. (2017), Sierra-Olivares, Prieto-Ayuso, Pastor-Vicedo, \& GonzálezVíllora (2020). Dicha línea muestra una serie de propuestas prácticas en el aprendizaje del fútbol base que no da respuesta a cómo el futbolista genera diferentes niveles de pensamiento cuando resuelve un problema en el juego, ni a la naturaleza de su toma de conciencia para construir el aprendizaje. Partiendo de este marco teórico, se ha profundizado en el desarrollo evolutivo que llevan a cabo los niños y los distintos tipos de pensamiento que se pueden generar, según las diferentes categorías de juego, aspecto novedoso hasta la fecha. Mostrando que para aprender en el fútbol base se deben crear puentes de unión entre la teoría y la práctica para poder avanzar (Karmiloff-Smith, 1994).

Bajo este telón de fondo se ha planteado un marco teórico adecuado para diseñar entrenamientos donde predomine el aprendizaje significativo bajo el paradigma cognitivo, teorías constructivistas, perspectiva funcional-estructural, siguiendo la línea marcada por autores como Del Valle (1999); Del Valle et al. (2008); De la Vega (2002); De la Vega et al. (2008), Rychecki, (2001) y Swartz et al. (2013), que contemplen tanto el desarrollo evolutivo que experimenta el niño, en función de la categoría de juego, utilizando diferentes estrategias para enseñar a pensar y así generar, partiendo de su conocimiento previo, distintos tipos de pensamiento en los jugadores.

Finalmente podemos añadir que en los entrenamientos simplemente con seguir una línea que albergue un proceso de aprendizaje con contenidos técnico-tácticos propios del fútbol, no es suficiente para determinar si el futbolista piensa $\mathrm{y}$ adquiere los conceptos necesarios para resolver jugadas efectivas y eficientes.
Se pone de manifiesto que en el aprendizaje del fútbol base se deben establecer uniones entre las etapas de desarrollo evolutivo del jugador con los contenidos que se pretende que éstos adquieran, amén de generar una metodología de acción eficaz para establecer un aprendizaje significativo utilizando el conflicto cognitivo.

El paradigma cognitivo, teorías constructivistas, perspectiva funcional-estructural establece un marco teórico de calidad que tiene en cuenta tanto la etapa de desarrollo evolutivo en la que se encuentra el niño; así como los diferentes tipos de pensamiento que genera el futbolista a la hora de solucionar situaciones creadas durante el juego, de manera eficaz.

\section{Futuras Líneas de Investigación}

Esta propuesta al ser novedosa, presenta varias líneas de investigación, las principales y más básicas radican por un lado en la necesidad de sumergirse más en el tema expuesto; sobre todo en los niveles de pensamiento que alcanzan los futbolistas a la hora de resolver situaciones de juego real, de tal forma que se amplíe el corpus teórico, con ejemplos concretos, cuyo objetivo sea crear metodologías que permitan un desarrollo de la enseñanza y un aprendizaje con más calidad.

Por otro lado centrar la atención en el entrenador y su formación permanente, de modo que se dé el proceso de autorregulación del conocimiento enfocado a la pericia y no al cúmulo de experiencia, permitiendo establecer puentes de unión entre la teoría y la práctica, para conseguir en el menor tiempo posible el mayor rendimiento.

Finalmente se debería extender esta nueva perspectiva teórico-práctica a otros deportes de invasión y por extensión al resto de ellos, para poder conseguir el mayor rendimiento en los deportistas en el menor tiempo posible, de forma eficiente y eficaz.

\section{Referencias}

Allard, F., Graham, S. \& Paarsalu, M. E. (1980). Perception in sport: Basketball. Journal of sport psychology, 2(1), 14-21.

Arda, T. \& Casal, C. (2003). Metodología de enseñanza del fútbol. Barcelona: Paidotribo.

Bard, C. \& Fleury, M. (1981). Considering eye movement as a predictor of attainment. En I. M. Cockerrill y W. W. Mc Cillivary (Eds.), Vision and sport 28-41. Cheltenham England: Stanley Thornes.

Benedek, E. (2001). Fútbol infantil. Barcelona: Paidotribo.

Bruggemann, D. (2004). Fútbol. Entrenamiento para niños y jóvenes. Barcelona: Paidotribo.

Bueno, M. L., Del Valle, S., De la Vega, R., Velázquez, R., González, J. M. \& Juárez, D. (2014). Modelo Perceptivo Motor a lo largo de todo el ciclo vital. Barcelona: Onporsport.

Cantos, J., Moreno, F. J. (2019). Pedagogía no lineal como método de enseñanza de los comportamientos tácticos en los deportes de equipo, aplicación al rugby. Revista Retos. 35, 402-406.

Corbeau, J. (1990). Fútbol. De la escuela a las asociaciones 
deportivas. Lérida: Agonos.

Corral, A. y Pardo, P. (1997). Psicología Evolutiva I (volumen 1). Madrid: UNED.

De la Vega, R. (2002). Desarrollo del metaconocimiento táctico y comprensión del juego: un enfoque constructivista aplicado al fútbol. Tesis Doctoral sin publicar. Madrid: UAM.

De la Vega, R., Del Valle, S., Maldonado, A. \& Moreno, A. (2008). Pensamiento y Acción en el Deporte. Perspectiva Funcional-Estructural. Sevilla: Wanceulen.

Del Valle, S. \& de la Vega, R. (2008). La regulación de la representación en los modelos emergentes en el Deporte. Perspectiva cognitiva. Retos. Nuevas tendencias en Educación Física, Deporte y Recreación, (13), 19-27.

Del Valle, S. (1999). La autorregulación de una tarea motriz: el equilibrio invertido. Tesis Doctoral sin publicar. Madrid: UAM.

Delval, J. (1994). El juego. El desarrollo humano. Madrid: Siglo XXI.

Fradua, L. (2005). El entrenamiento con jóvenes jugadores en las escuelas de fútbol. Documento inédito. Máster Universitario de Preparación Física en Fútbol. RFEFUCLM.García Aliaga, A. (2005). Entrenamiento táctico en el fútbol base: fundamentos metodológicos y conceptuales. Máster Universitario de Preparación Física en Fútbol. RFEF-UCLM.

Garganta, J. \& Pinto, J. (1997). La enseñanza del fútbol. En Graça, A. y Oliveira, J. (Coord.) La enseñanza de los juegos deportivos. Paidotribo. Barcelona.

Garganta, J., \& Cunha e Silva, P. (2000). O jogo de futebol: entre o caos e a regra. Revista Horizonte, 16(91), 5-8.

Gil,A., Del Villar, F., Claver, F., Moreno,A., García, L., Moreno, M. P. (2012). ¿Existe relación entre el nivel de competición y el conocimiento de juego en voleibol? Revista Retos 21,53-57.

Karmiloff-Smith, A. (1994). Más allá de la modularidad. Madrid: Alianza Editorial.

Lacasa, P. \& Villuendas, D. (1988). Acción y representación en el niño: Interacción social y aprendizaje. Madrid: CIDE.

Lago, C. (2001). El proceso de iniciación en el fútbol. Secuenciación de los contenidos técnico-tácticos. Training Fútbol, 6, 34-45.

Lago, C. (2002). La enseñanza del fútbol en la edad escolar. Sevilla: Wanceulen.

Lago, C. (2007). Planificación de los contenidos técnicotácticos individuales y grupales del fútbol en las categorías benjamín, infantil y cadete. Ponencia del módulo 8 Metodología de calidad de la enseñanza del fútbol. Documento inédito. Master detección y formación del talento en jóvenes futbolistas. Madrid.

Lealli, G. (1994). Fútbol Base. Entrenamiento óptimo del futbolista en el período evolutivo. Barcelona: Martínez Roca.

Martínez Garfia, F. \& Sáenz-López Buñuel, P. (2000). Iniciación al Fútbol, Sevilla: Wanceulen.

Moreno, R. \& Morcillo, J.A. (2004). La enseñanza del fútbol en las escuelas deportivas de iniciación. Madrid: Gymnos.

Pacheco, R. (2004). La enseñanza y el entrenamiento del fútbol 7. Un juego de iniciación al fútbol 11. Barcelona: Paidotribo.

Piaget, J. (1974/1985b). La toma de conciencia. Madrid: Morata.

Piaget, J. (1976). The graps of consciousness: Action and concept in the young child. Cambridge, MA: Harvard University Press.

Rychecki, F. (2001). El conocimiento de la acción final en judo a través de los diferentes canales de comunicación. Tesis doctoral sin publicar. Madrid: UAM.

Sampedro, J. (2000). Fundamentos de táctica deportiva. Análisis de la estrategia de los deportes. Madrid: Gymnos.

Seirul-lo, F. (2004). Estructura socioafectiva, Master Profesional en Alto Rendimiento en Deportes de Equipo: Área Coordinativa. Documento inédito. Barcelona: Byomedic - Fundació F.C. Barcelona.

Sierra-Olivares, J., García-Rubio, J. (2017). La problemática táctica, clave en el diseño representativo de tareas desde el enfoque de la pedagogía no lineal aplicada al deporte. Revista Retos. 32, 270-278.

Sierra-Olivares, J., Prieto-Ayuso- A., Pastor-Vicedo, J.C., \& González-Víllora, S. (2020). Proposal for a multidisciplinary assessment of talented football players. Revista Retos, pendiente de publicación.

Solá, J. (1998). Formación cognoscitiva y rendimiento táctico. Apunts, 3(53), 33-41.

Solá, J. (2005). Estudio funcional del saber deportivo para la comprensión táctica. Apunts, 4(82), 26-35.

Suárez, P. \& Hernández, A. (2007). Aprendizaje motor: una breve revisión teórica. EF Deportes. Revista Digital, 109, $1-17$.

Swartz, R., Costa, A., Beyer, B., Reagan, R., \& Kallick, B. (2013). El aprendizaje basado en el pensamiento. España: SM.

Vegas Haro, G. (2006). Metodología de enseñanza basada en la implicación cognitiva del jugador de fútbol base. Tesis Doctoral sin publicar. Granada: Universidad de Granada.

Vuyk, R. (1981). Overview and Critique of Piaget's Genetic Epistemology. 1965-1980. (Vols. 2). Nueva York: Academic Press. (Trad. cast. de C. del Barrio y A. Corral. Panorámica y crítica de la epistemología genética de Piaget 1965-1980. Vol I y II. Madrid: Alianza, 1984/1986).

Zamora, J. L. (2006). Los paradigmas curriculares de la Educación Física. Actas del II Congreso Boliviano Internacional de Currículo. Educación Física y Deporte Escolar: Venezuela.

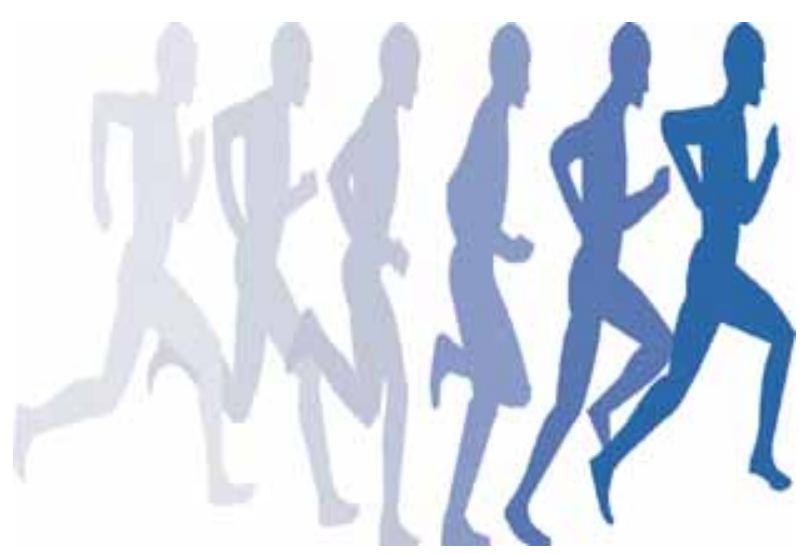

